\title{
Evaluation of the Electromagnetic Power Absorption in Humans Exposed to Plane Waves: The Effect of Breathing Activity
}

\author{
Marta Cavagnaro, Erika Pittella, and Stefano Pisa \\ Department of Information Engineering, Electronics and Telecommunications, Sapienza University of Rome, 00184 Rome, Italy \\ Correspondence should be addressed to Erika Pittella; pittella@diet.uniromal.it
}

Received 28 June 2013; Accepted 27 November 2013

Academic Editor: Andrea Borsic

Copyright ( 2013 Marta Cavagnaro et al. This is an open access article distributed under the Creative Commons Attribution License, which permits unrestricted use, distribution, and reproduction in any medium, provided the original work is properly cited.

\begin{abstract}
The safety aspects of the exposure of people to uniform plane waves in the frequency range from $900 \mathrm{MHz}$ to $5 \mathrm{GHz}$ are analyzed. Starting from a human body model available in the literature, representing a man in resting state, two new anatomical models are considered, representing different phases of the respiratory activity: tidal breath and deep breath. These models have been used to evaluate the whole body Specific Absorption Rate (SAR) and the 10-g averaged and 1-g averaged SAR. The analysis is performed using a parallel implementation of the finite difference time domain method. A uniform plane wave, with vertical polarization, is used as an incident field since this is the canonical exposure situation used in safety guidelines. Results show that if the incident electromagnetic field is compliant with the reference levels promulgated by the International Commission on Non-Ionizing Radiation Protection and by IEEE, the computed SAR values are lower than the corresponding basic restrictions, as expected. On the other side, when the Federal Communications Commission reference levels are considered, 1-g SAR values exceeding the basic restrictions for exposure at $4 \mathrm{GHz}$ and above are obtained. Furthermore, results show that the whole body SAR values increase passing from the resting state model to the deep breath model, for all the considered frequencies.
\end{abstract}

\section{Introduction}

The fast developments of wireless communications systems, such as Universal Mobile Telecommunications System (UMTS), WiFi, and Worldwide Interoperability for Microwave Access (WiMAX), have increased the spectrum of fields radiated by the fixed stations of these systems to frequencies up to $5 \mathrm{GHz}$ [1]. Moreover, biomedical applications of electromagnetic fields at microwave frequencies, as the remote monitoring of vital signs, are gaining increasing attention $[2,3]$. Therefore, the study of human exposure at frequencies between $900 \mathrm{MHz}$ and $5 \mathrm{GHz}$ is an up-to-date topic in order to assess the compliance of the electromagnetic field with exposure guidelines [4-7].

Exposure guidelines are defined in terms of basic restrictions, which are maximum values directly linked to health effects, and in terms of reference levels, which are values related to the incident electromagnetic fields more easily measurable in actual applications [4-7]. In the considered frequency range the basic restrictions are defined in terms of the Specific Absorption Rate (SAR) as averaged over the whole body or over a local mass [4-7]. From the exposure guideline point of view, an important change has occurred within the 2005 version of the IEEE exposure standard [6] with respect to the previous versions [8]. In particular, for local exposure evaluation, the IEEE 2005 has adopted a limit on the 10-g averaged SAR, similarly to the ICNIRP basic restriction, in place of the limit on the 1-g averaged SAR present in the preceding versions of the Standard [8]. Moreover, the ICNIRP reference levels for the unperturbed incident field have been also adopted [6]. However the 1-g averaged SAR is still implemented by the Federal Communications Commission (FCC) regulation [7].

In the last decade the progress in computer resources has made the numerical studies of human exposure to electromagnetic fields feasible for anatomically realistic body models and for frequencies up to $1 \mathrm{GHz}$ and above [912]. Moreover several numerical models of the human body have been developed and studied [13-17]. However, all the reported studies have considered human models with 
the lung at resting state. On the other side, the development of anatomical models of breathing subjects could be very helpful to evaluate safety issues related to newly developed diagnostic techniques. As an example, Ultra Wideband Radars for the remote monitoring of the human respiratory activity are spreading in recent years.

In this paper, three human body models have been considered. Starting from Duke of the Virtual Family [13], two new anatomical models have been developed taking into account the respiration physiology and the pulmonary mechanics. These new models, representing a tidal and a deep breath, have been obtained through an automatic procedure [18]. The exposure of these three different breathing adult models to an incident electromagnetic field with frequencies in the range $900 \mathrm{MHz}-5 \mathrm{GHz}$ is studied by using a parallel implementation of the finite difference time domain (FDTD) method. The whole body SAR, the SAR as averaged on 10-g mass and 1-g mass $\left(\mathrm{SAR}_{\mathrm{WB}}, \mathrm{SAR}_{10-\mathrm{g}}\right.$, and $\left.\mathrm{SAR}_{1-\mathrm{g}}\right)$ are computed, taking into account the frequency dependence of the dielectric properties of biological tissues, and also the variations of the dielectric properties and mass density of the lungs during respiration.

The paper is organized as follows: in Section 2, the numerical method and the human anatomical models are presented; in Section 3 results of dosimetry studies for the three breathing subject models are shown in terms of SAR. Eventually, in Section 4 conclusions are drawn.

\section{Methods and Models}

2.1. FDTD Method. The SAR computations are performed by using a self-developed code [19]. This code applies the FDTD method that divides the domain under study in elementary cells. To excite the impinging plane wave the whole volume is divided into a total-field and a scattered-field region by means of a Huygens' surface [20, 21]. The scattered-field region is closed by applying a uniaxial perfectly matched layer (UPML) absorbing boundary condition made by 5 cells with a parabolic spatial dependence for the electric and magnetic conductivities, and whose maximum values give a theoretical reflection coefficient lower than $0.01 \%$ [21]. A spatial resolution of $1 \mathrm{~mm}$ was chosen for the simulations. At the highest frequency of interest $(5 \mathrm{GHz})$ this resolution corresponds to less than $1 / 8$ of the wavelength for all body tissues and it is almost equal to $1 / 10$ of the penetration depth of the electromagnetic field. In [12] an expanded uncertainty of $0.2 \mathrm{~dB}$ on the $S A R_{W B}$ and of $0.51 \mathrm{~dB}$ for the $S A R_{10-\mathrm{g}}$ was evaluated for 1-mm FDTD simulations taking into account grid resolution, absorbing boundary conditions, and steadystate convergence.

With reference to the source, a plane wave with sinusoidal time dependence propagating along the $y$-axis (front-back of the body model) and with the electric field polarized along the $z$-axis (feet-head direction of the body model) has been excited with an electric field amplitude corresponding to the ICNIRP and IEEE 2005 reference levels for general public at the different frequencies. For the 1-g averaged SAR the reference levels for general public of FCC have been considered instead.

The $1-\mathrm{mm}$ resolution of the human body model requires a huge amount of memory (higher than 15 GB) and computational time (several days). For this reason, a parallel implementation of the FDTD scheme based on the message passing interface (MPI) paradigm was used. Simulations were run on a cluster with a total of fifteen CPUs and about 26 GB of RAM. Execution time is about 8 hours for each considered model. The $S A R_{10-g}$ and $S A R_{1-g}$ have been computed by considering cubic volumes, whose sides are expanded until the desired mass is obtained, allowing a maximum $10 \%$ of air inclusion.

2.2. Human Breathing Models. As regards the human body models, the FDTD method requires an anatomical model divided into cells with different properties (voxel). Starting from an anatomical model available in the literature, new models of the human anatomy have been developed to represent different phases of the respiratory activity, taking into account the respiration physiology and the pulmonary mechanics [22]. In particular, the body models usually considered in dosimetry studies represent a resting state body (RS), that is a body at the end of the respiration phase, when little air is present into the lungs. In [18], through an automatic procedure, two new models have been developed from Duke of the Virtual Family. In brief, starting from the RS human model, constituted by a three-dimensional matrix of integer numbers representing the various tissues (77 tissues), lung cells have been added simulating both a diaphragm displacement and an enlargement of the rib cage. Changing the entity of the diaphragm displacement and of the rib cage enlargement, two respiration phases have been modelled: the tidal breath (TB), corresponding to a state of normal inspiration with an inhaled volume of air equal to about $500 \mathrm{~mL}$, and the deep breath (DB), corresponding to a state in which the volume of inhaled air is increased by $860 \mathrm{~mL}$. It is important to note that the DB case refers to a deeper respiration with respect to the tidal one, as expected for a subject during normal daily activities [22], and it does not correspond with the Inspiration Reserve Volume that is more than two times the RS.

Figure 1 shows the number of lung cells in each horizontal section of the human body, as a function of the height from the feet, for the three human body models considered: resting state, that is the original model, tidal breath, and deep breath. From the figure, an overall view of the levels where the lung expansion occurred is obtained. In particular, it can be noted that the rib cage expansion in the TB and DB models follows the behavior of the RS model in the higher sections (from $140 \mathrm{~cm}$ to $150 \mathrm{~cm}$ in Figure 1). Then the expansion becomes more irregular; this is due to the drop in the diaphragm of $2 \mathrm{~cm}$ and $4 \mathrm{~cm}$ for the TB model and DB model, respectively.

The two new lung models are anatomically consistent. Moreover, they have a final volume of $4262 \mathrm{~mL}$ and $4530 \mathrm{~mL}$, for $\mathrm{TB}$ and $\mathrm{DB}$, respectively. Since the initial volume was $3849 \mathrm{~mL}$ (RS), an increase in volume of about $413 \mathrm{~mL}$ and $681 \mathrm{~mL}$ has been found for TB and DB, respectively, in agreement with [22]. Figure 2 shows a $2 \mathrm{D}$ axial section at 


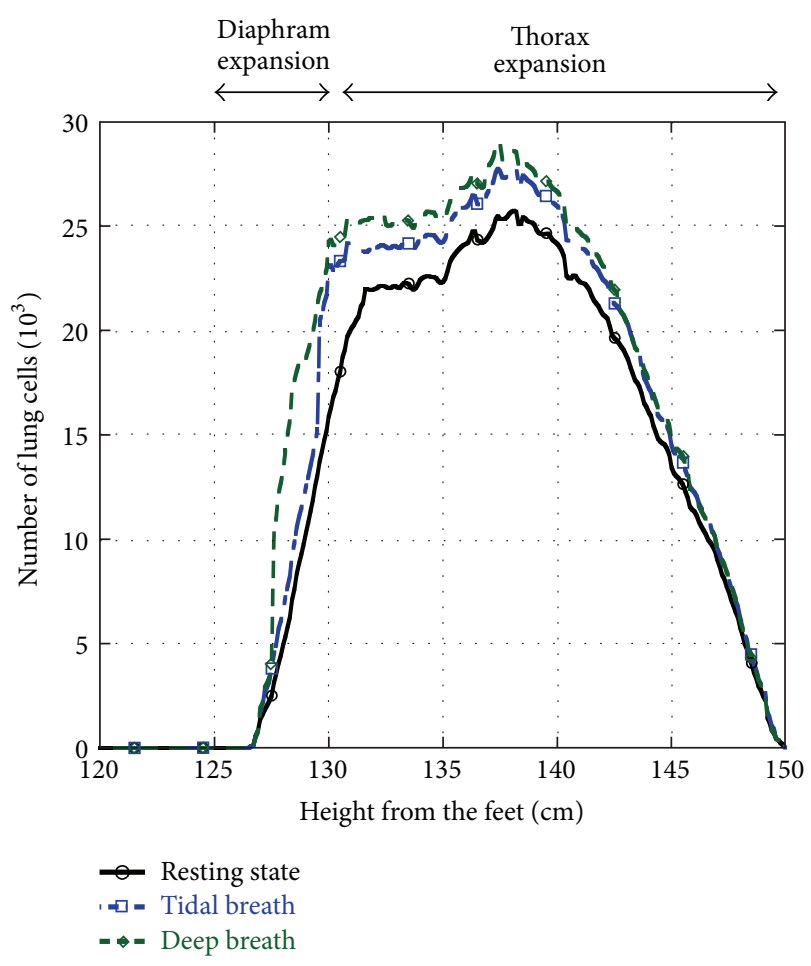

FIGURE 1: Number of lung cells, in the transversal plane, for the RS, $\mathrm{TB}$, and $\mathrm{DB}$ models, as a function of the height from the feet.

the level of the diaphragm, for the RS ad DB model. The figure evidences that in the DB model the diaphragm is not present anymore at this level due to its downward displacement.

In the simulations the values reported in the data base, available online [23], have been assigned to the dielectric parameters of the 77 tissues comprising the body models, taking into account the frequency dependence.

With reference to the lung, for the breathing models (TB and DB models), the mass density has been assumed equal to that of inflated lung $\left(\rho_{\mathrm{LI}}=394 \mathrm{~kg} / \mathrm{m}^{3}\right)$ [23], while for the RS model (man at the end of the expiration phase) an average value between the inflated lung $\left(\rho_{\mathrm{LI}}\right)$ and deflated lung mass density $\left(\rho_{\mathrm{LD}}=1050 \mathrm{~kg} / \mathrm{m}^{3}\right)$ has been used, since the lung is never totally deflated because of the dead space. As concerns the dielectric properties, the values for inflated lungs have been used for TB and DB models. At resting state, an average value between those of inflated and deflated lung has been used, according to the linear dependence of lung dielectric properties from the air volume filling factor [24].

\section{Results}

The obtained models have been exposed to a plane wave with frequencies between $900 \mathrm{MHz}$ and $5 \mathrm{GHz}$.

When the $\mathrm{SAR}_{\mathrm{WB}}$ and $\mathrm{SAR}_{10-\mathrm{g}}$ have been evaluated, the incident power was defined equal to the ICNIRP and IEEE2005 reference levels for general public; when the $\mathrm{SAR}_{1-\mathrm{g}}$ has been considered, the incident power was settled equal to the FCC reference levels.
3.1. SAR Whole Body. $\mathrm{SAR}_{\mathrm{WB}}$ values obtained in the Duke's breathing models as a function of frequency are depicted in Figure 3, together with the power density reference level allowed for general public in ICNIRP and IEEE 2005 guidelines $[4,6]$. Results point out an increase in the $\mathrm{SAR}_{\mathrm{WB}}$ with the frequency up to $2 \mathrm{GHz}$ and a decrease above this frequency value.

However it is worth noting that this behavior is strictly due to the limits on the power density settled in the guidelines, which increase with the frequency up to $2 \mathrm{GHz}$ and are maintained constant for higher frequencies, as clearly shown in Figure 3. Indeed a $S_{A R}$ linearly decreasing with the frequency would be obtained if the power density is maintained constant for all the frequencies. From Figure 3 it can be noted that all the considered models lead to $\mathrm{SAR}_{\mathrm{WB}}$ values compliant with the basic restrictions for all the considered frequencies. In particular, a very little difference is obtained in the values of the $\mathrm{SAR}_{\mathrm{WB}}$ for the three anatomical breathing models at all the considered frequencies, with increasing values of the absorbed power passing from the resting state model to the deep breath model. However, increasing the frequency, the difference in the whole body SAR for the three considered models decreases $\left(\mathrm{SAR}_{\mathrm{WB}}\right.$ @900 MHz: $6.50 \cdot 10^{-2} \mathrm{~W} / \mathrm{kg}(\mathrm{RS}), 6.54 \cdot 10^{-2} \mathrm{~W} / \mathrm{kg}(\mathrm{TB})$, $6.55 \cdot 10^{-2} \mathrm{~W} / \mathrm{kg}$ (DB); SAR $\mathrm{WB}_{\mathrm{WB}} @ 5 \mathrm{GHz}: 3.59 \cdot 10^{-2} \mathrm{~W} / \mathrm{kg}(\mathrm{RS})$, $3.71 \cdot 10^{-2} \mathrm{~W} / \mathrm{kg}$ (TB), $\left.3.74 \cdot 10^{-2} \mathrm{~W} / \mathrm{kg}(\mathrm{DB})\right)$.

Figure 4 shows the SAR distribution in a coronal plane for the resting state model at two different frequencies ( $900 \mathrm{MHz}$ and $5000 \mathrm{MHz}$ ). The figure evidences that the power absorption is strictly dependent on the frequency and that it remains superficially confined at the higher one.

3.2. $S A R_{10-g}$. Figure 5 shows the peak values of the $S A R_{10-g}$ obtained in the head and trunk for the three considered body models, together with the corresponding ICNIRP and IEEE 2005 basic restriction. In the figure the incident power density allowed in ICNIRP and IEEE 2005 is also reported.

The figure shows an increase with the frequency of the obtained values up to $2.4 \mathrm{GHz}$, followed by a linear decrease. However, all the $\mathrm{SAR}_{10-\mathrm{g}}$ values are well below the corresponding basic restriction $(2 \mathrm{~W} / \mathrm{kg})$. It has to be noted here that the position where the maximum $\mathrm{SAR}_{10-\mathrm{g}}$ is obtained changes with the frequency, from nose (for all the three models at $900 \mathrm{MHz}$ ) to genital organs (for RS at $1800 \mathrm{MHz}$ and for all the three models at $2000 \mathrm{MHz}$ and $5000 \mathrm{MHz}$ ), thorax (for TB and DB at $1800 \mathrm{MHz}$ ), chin (RS and DB at $2400 \mathrm{MHz}$ ), and neck (for TB at $2400 \mathrm{MHz}$ ).

With reference to the different anatomical models, no meaningful differences have been obtained in the $\mathrm{SAR}_{10-\mathrm{g}}$ values. This is because these values are obtained mainly in the head and in the genital organs outside the area anatomically affected by the respiration. However, it is interesting to note that, at $1800 \mathrm{MHz}$, while the head-trunk maximum $\mathrm{SAR}_{10-\mathrm{g}}$ is found in the genital organs of the RS models, it is found in the breast in the other two body models. This result could be linked to the wider thorax of the TB and DB models, with respect to the RS one, obtained as a consequence of the expansion of the lung volumes. 


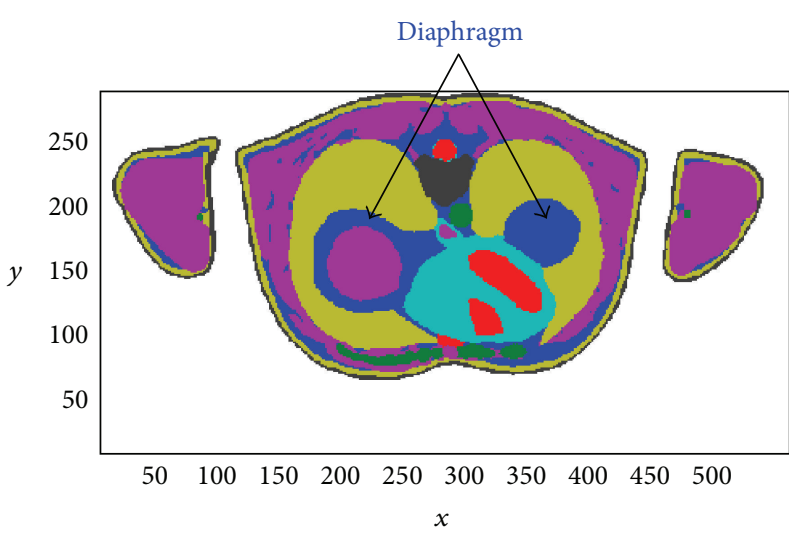

(a)

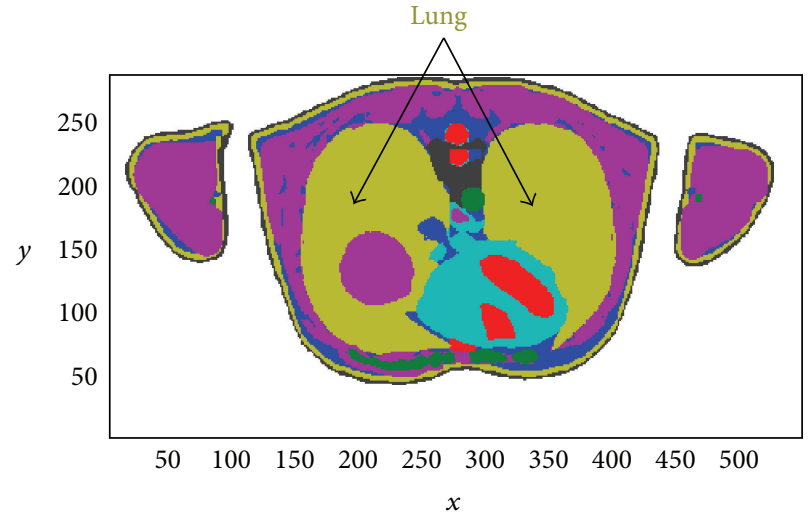

(b)

Figure 2: Bidimensional representation of an axial section $(z=130 \mathrm{~cm})$ for the RS (a) and DB (b) model.

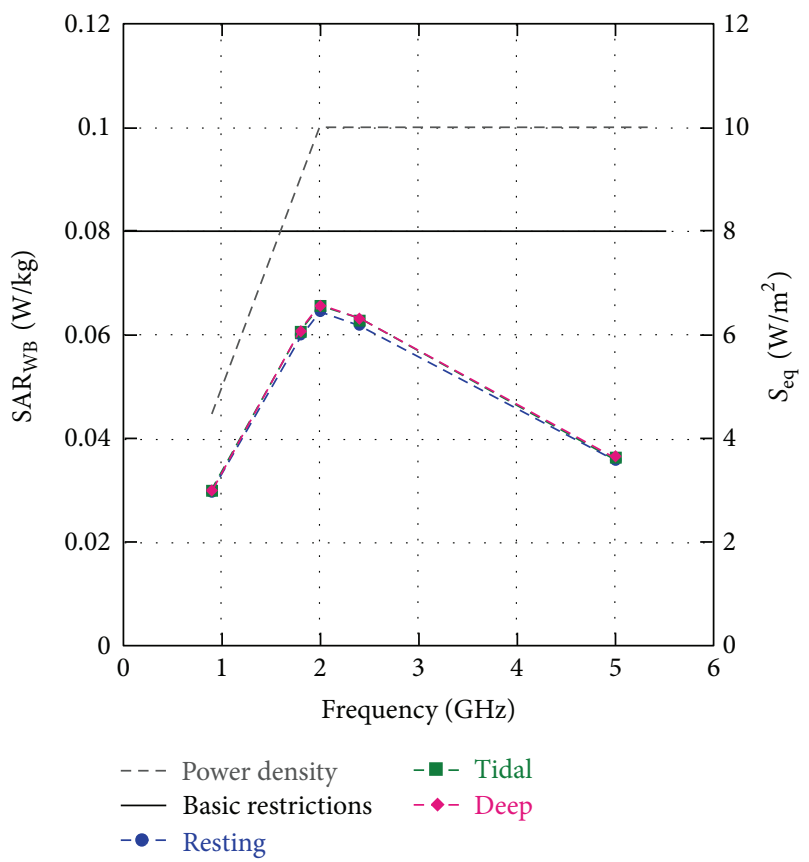

Figure 3: Whole body SAR for the RS, TB, and DB models and the input power density reference level allowed for general public in ICNIRP and IEEE 2005 guidelines.

3.3. $S A R_{10-g}$ in the Lung. With reference to the lungs, the $S_{A R}$-g values obtained for this particular tissue have been drawn in Figure 6 as a function of the frequency, for the three considered models. In the figure the power density reference levels, allowed for general public in ICNIRP and IEEE 2005 guidelines and used for the incident plane wave, are also reported.

From the figure it is clearly visible that the absorbed power increases passing from the resting state model to the deep model and that the higher difference occurs at $900 \mathrm{MHz}$ (with a percentage variation of $76.77 \%$ ), where the $S_{A} R_{10-g}$ moves from the value of $0.063 \mathrm{~W} / \mathrm{kg}$ for the resting state to $0.110 \mathrm{~W} / \mathrm{kg}$ for the deep breath model. This effect is due to the decrease of the lung mass density from the deflated model (RS) to inflated ones (TB and DB).

3.4. $S A R_{1-g}$. Figure 7 shows the results related to the maximum $S_{1-g}$ in the head and trunk. The position of the maximum $\mathrm{SAR}_{1-\mathrm{g}}$ changes with the frequency, from the nose (for all the three models at $900 \mathrm{MHz}, 1800 \mathrm{MHz}$, and $2000 \mathrm{MHz}$ ) to the neck (for all the three models at $2400 \mathrm{MHz}$ ) and genital organs (for all the three models at $5000 \mathrm{MHz}$ ). From the figure it is interesting to note the differences in the maximum $\mathrm{SAR}_{1-\mathrm{g}}$ for the different body models. Moreover, it can be noted that the $\mathrm{SAR}_{1-\mathrm{g}}$ increases with the frequency above $2.4 \mathrm{GHz}$, even if the incident power does not increase any more, contrary to the other SAR results. This increase leads to a local SAR exceeding the basic restriction level of $1.6 \mathrm{~W} / \mathrm{kg}$ of the FCC regulation for frequencies above $4 \mathrm{GHz}$ for all the considered models. Similar results have been obtained in [12], where the computed $\mathrm{SAR}_{1-\mathrm{g}}$ values exceeded the basic restrictions of $1.6 \mathrm{~W} / \mathrm{kg}$ at frequencies above $1.5 \mathrm{GHz}$, both for IEEE-1999 and FCC reference levels, for different models of adult and child.

With reference to the lungs, the $S A R_{1-g}$ values obtained for this tissue have been drawn in Figure 8 as a function of the frequency, for the three considered models, together with the power density reference level allowed for general public in ICNIRP and IEEE 2005 guidelines.

From the figure it is clearly visible that the $\mathrm{SAR}_{1-\mathrm{g}}$ increases passing from the resting state model to the deep breath model.

\section{Conclusions}

This paper addresses the safety aspects of people exposed to uniform plane waves in the frequency range from $900 \mathrm{MHz}$ to $5 \mathrm{GHz}$. The problem has been analyzed using a parallel numerical code and considering three accurate models of breathing humans. These models have been developed through an automatic procedure starting from Duke's model available in the Virtual Family dataset. The human body models have been exposed to a plane wave with vertical 


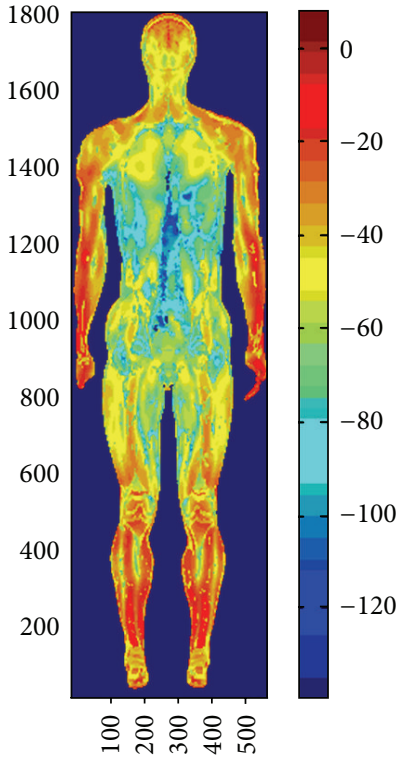

(a)

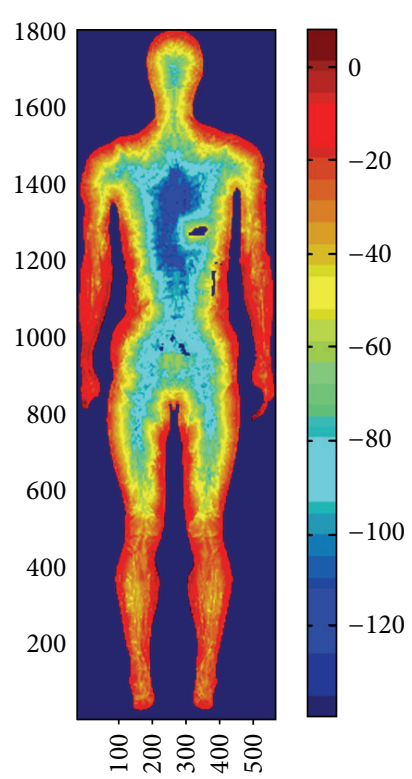

(b)

FIgURE 4: Whole body SAR $\left(\mathrm{dB}_{\mathrm{W} / \mathrm{kg}}\right)$ for the RS model at $900 \mathrm{MHz}$ (a) and at $5000 \mathrm{MHz}$ (b).

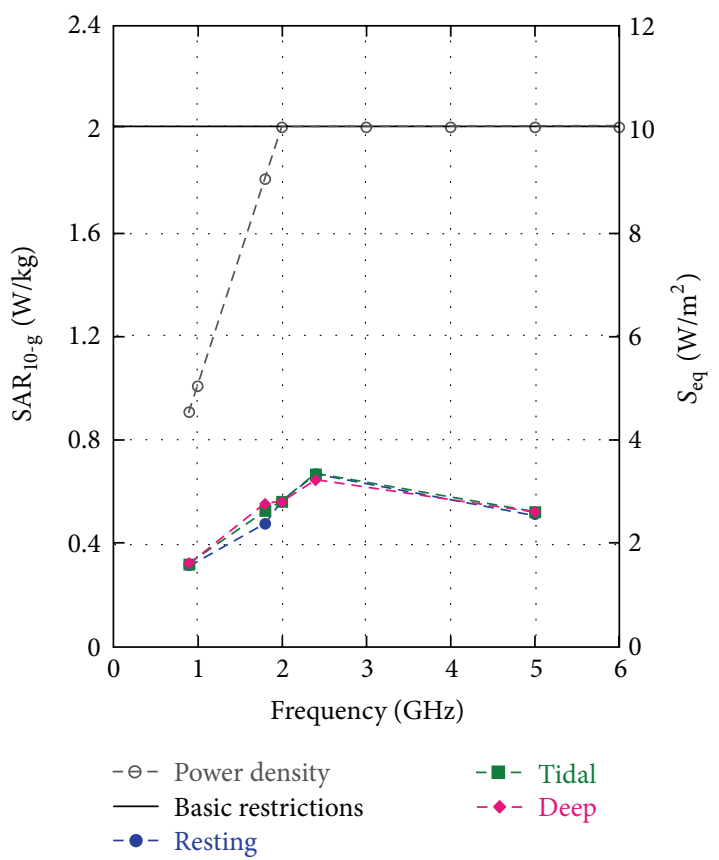

FIGURE 5: $\mathrm{SAR}_{10-\mathrm{g}}$ for the RS, TB, and DB models and the power density reference level allowed for general public in ICNIRP and IEEE 2005 guidelines.

polarization and with incident power densities equal to those settled by the safety guidelines for the different frequencies considered.

Results show that for all the considered frequencies and body models the $\mathrm{SAR}_{\mathrm{WB}}$ and $\mathrm{SAR}_{10 \text {-g }}$ do not exceed the $0.08 \mathrm{~W} / \mathrm{kg}$ and $2 \mathrm{~W} / \mathrm{kg}$ basic restrictions. Moreover, $\mathrm{SAR}_{\mathrm{WB}}$ decreases with the frequency if the incident power density is

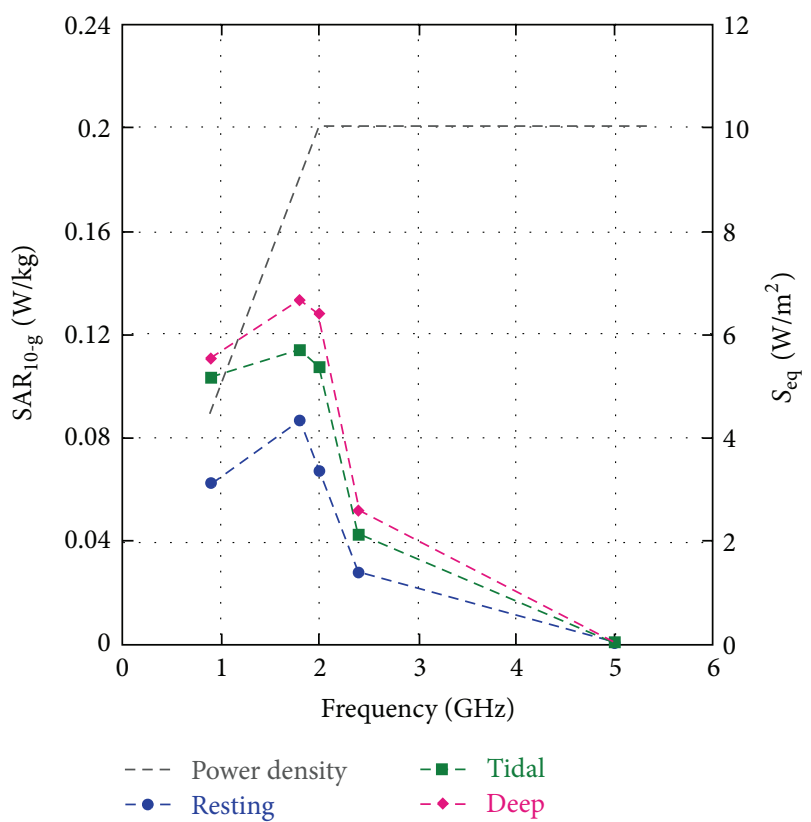

FIGURE 6: $\mathrm{SAR}_{10 \mathrm{~g}}$ in the lungs for the RS, TB, and DB models and the power density reference level allowed for general public in ICNIRP and IEEE 2005 guidelines.

maintained constant and it is the most stringent parameter compared to $\mathrm{SAR}_{10-\mathrm{g}}$, for assessing the exposure compliance. On the other side, the $\mathrm{SAR}_{1-\mathrm{g}}$ increases with the frequency and local $S_{1-g}$ values exceeding the basic restriction of $1.6 \mathrm{~W} / \mathrm{kg}$ are obtained above $4 \mathrm{GHz}$ when the incident power density is settled at the FCC reference levels. From Figures 3,5 , and 7 it is interesting to note that the $\mathrm{SAR}_{\mathrm{WB}}$ shows 


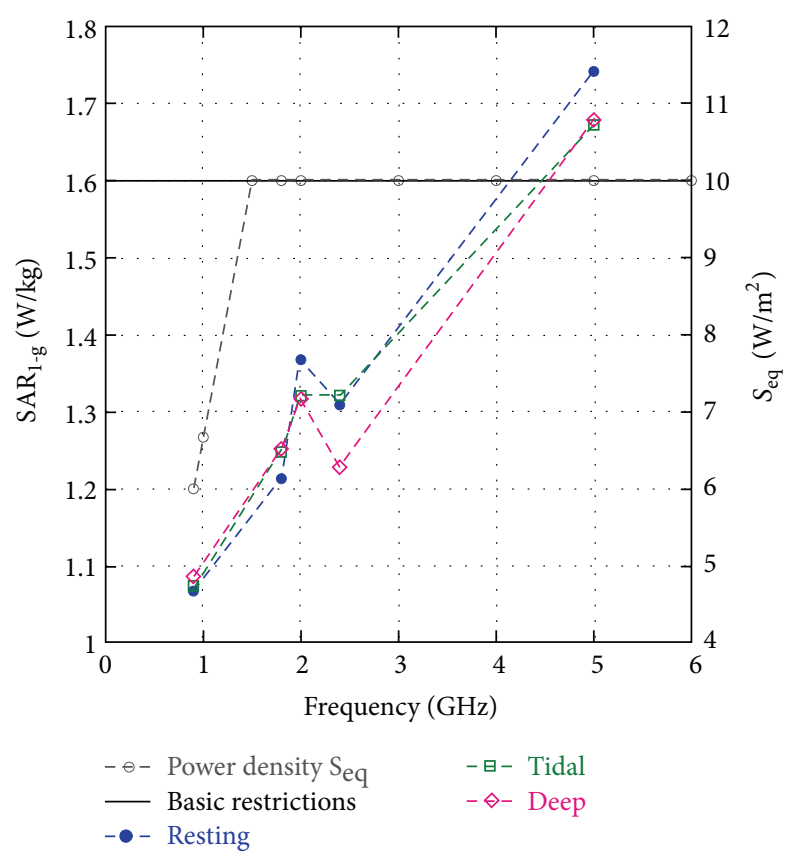

FIGURE 7: $\mathrm{SAR}_{1-\mathrm{g}}$ for the RS, TB, and DB models for FCC reference levels. The FCC power density reference level allowed for general population is also reported.

the maximum value in correspondence of the frequency in which the maximum incident power density is allowed by the safety guidelines, while the $\mathrm{SAR}_{10-\mathrm{g}}$ and the $\mathrm{SAR}_{1-\mathrm{g}}$ maximum are obtained at higher frequencies. This different behavior is due to the fact that while the $\mathrm{SAR}_{\mathrm{WB}}$ is directly linked to the total power impinging on the whole body model, the local SARs have different averaging volumes and are influenced by the field penetration depth that in turn depends on the electrical properties of the different tissues involved. Comparing the three body models, for all the considered frequencies, the SAR values are higher in the body model representing a deep respiration with respect to the model representing a resting state for the $S A R_{W B}$ and $S A R_{10-g}$.

To conclude it is worth mentioning that the obtained results are based on specific body models developed from an adult male model and considering the dielectric properties values available in the literature [23]. With reference to the influence of the uncertainty of the dielectric properties values on the obtained SAR results, in [25] an extensive analysis has been conducted changing the dielectric properties values of the 39 tissues comprising a $3-\mathrm{mm}$ resolution human body model and considering plane-wave irradiation at the frequencies of 200, 400, 918, and $2060 \mathrm{MHz}$. They found that the changing values did not substantially influence the $S A R_{W B}$, while a higher influence was obtained on localized SAR values for the individual tissues where the properties were changed. Finally, in $[26,27]$ a marginal effect of the variation of dielectric properties on the $\mathrm{SAR}_{10-\mathrm{g}}$ was obtained (less than 10\%). With reference to the body models used, further studies should be performed in order to confirm the obtained results considering different subject anatomies,

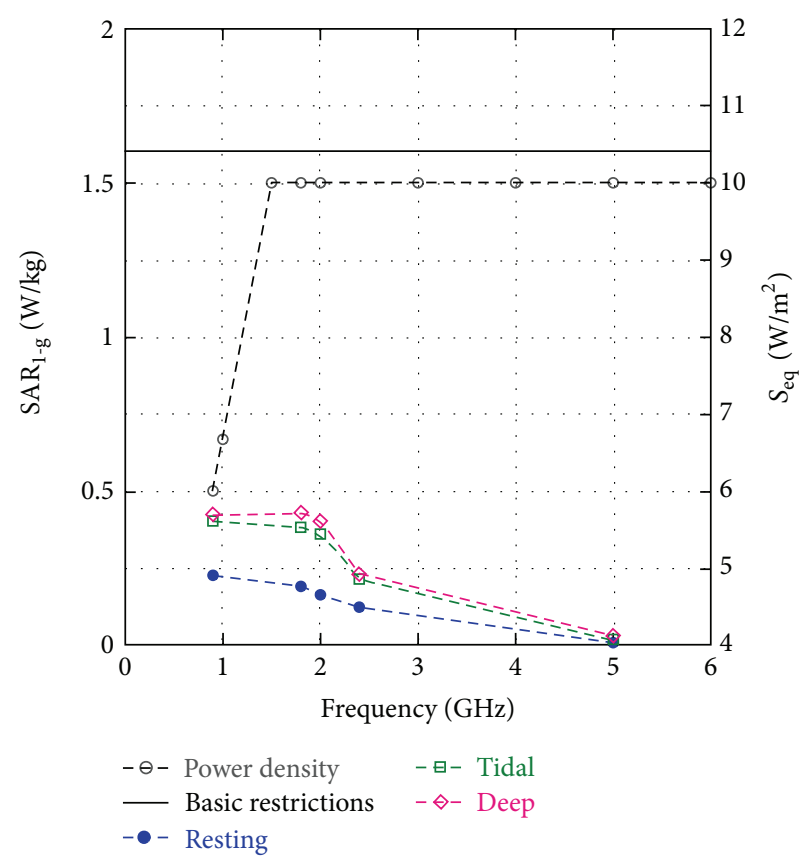

FIGURE 8: $\mathrm{SAR}_{1-\mathrm{g}}$ in the lungs for the RS, TB, and DB models obtained for FCC reference levels. The FCC power density reference level allowed for general population is also reported.

women, and children as, for example, those developed by IT'IS Foundation. In particular, the automatic procedure used to develop the TB and DB body models could be applied to obtain breathing women and children. These models can be used both to study the absorption associated to exposure to the electromagnetic field emitted by communication systems and by biomedical applications of electromagnetic fields [28] and to characterize the human anatomy to be used in the development of new biomedical systems [29]. Furthermore, these models can be used to compute the Radar Cross Section (RCS) of man during respiration; this information is of great importance for ultrawideband radar design.

\section{References}

[1] S. Allen, J. C. Lin, J. Bach Anderson et al., "ICNIRP statement on EMF-emitting new technologies," Health Physics, vol. 94, no. 4, pp. 376-392, 2008.

[2] E. M. Staderini, "UWB radars in medicine," IEEE Aerospace and Electronic Systems Magazine, vol. 17, no. 1, pp. 13-18, 2002.

[3] E. Pittella, P. Bernardi, M. Cavagnaro, S. Pisa, and E. Piuzzi, "Design of UWB antennas to monitor cardiac activity," Applied Computational Electromagnetics Society Journal, vol. 26, no. 4, pp. 267-274, 2011.

[4] ICNIRP, "ICNIRP statement on the guidelines for limiting exposure to time-varying electric, magnetic, and electromagnetic fields (UP to $300 \mathrm{GHz}$ )," Health Physics, vol. 97, no. 3, pp. 257-258, 2009.

[5] Commission of European Communities, Ed., Council Recommendation on Limits For Exposure of the General Public To Electromagnetic Fields: $0 \mathrm{~Hz}-300 \mathrm{GHz}$, EC, Brussels, Belgium, 1998. 
[6] IEEE Standard for Safety Levels with Respect to Human Exposure to Radio Frequency Electromagnetic Fields, $3 \mathrm{kHz}$ to $300 \mathrm{GHz}$, IEEE Standard C95. 1, 2005.

[7] Federal Communications Commission Office of Engineering \& Technology, "Evaluating compliance with FCC guidelines for human exposure to radiofrequency electromagnetic fields," OET Bulletin 65, Washington, DC, 1997.

[8] IEEE Standard for Safety Levels with Respect to Human Exposure to Radio Frequency Electromagnetic Fields, $3 \mathrm{kHz}$ to $300 \mathrm{GHz}$, IEEE Standard C95. 1, 1999.

[9] P. Bernardi, M. Cavagnaro, S. Pisa, and E. Piuzzi, "SAR distribution and temperature increase in an anatomical model of the human eye exposed to the field radiated by the user antenna in a wireless LAN," IEEE Transactions on Microwave Theory and Techniques, vol. 46, no. 12, pp. 2074-2082, 1998.

[10] L. Sandrini, A. Vaccari, C. Malacarne, L. Cristoforetti, and R. Pontalti, "RF dosimetry: a comparison between power absorption of female and male numerical models from 0.1 to 4 GHz," Physics in Medicine and Biology, vol. 49, no. 22, pp. 51855201, 2004.

[11] P. Bernardi, M. Cavagnaro, S. Pisa, and E. Piuzzi, "Specific absorption rate and temperature elevation in a subject exposed in the far-field of radio-frequency sources operating in the 10900-MHz range," IEEE Transactions on Biomedical Engineering, vol. 50, no. 3, pp. 295-304, 2003.

[12] E. Piuzzi, P. Bernardi, M. Cavagnaro, S. Pisa, and J. C. Lin, "Analysis of adult and child exposure to uniform plane waves at mobile communication systems frequencies $(900 \mathrm{MHz}-3$ $\mathrm{GHz}$ )," IEEE Transactions on Electromagnetic Compatibility, vol. 53, no. 1, pp. 38-47, 2011.

[13] A. Christ, W. Kainz, E. G. Hahn et al., "The virtual familydevelopment of surface-based anatomical models of two adults and two children for dosimetric simulations," Physics in Medicine and Biology, vol. 55, no. 2, pp. N23-N38, 2010.

[14] T. Nagaoka, S. Watanabe, K. Sakurai et al., "Development of realistic high-resolution whole-body voxel models of Japanese adult males and females of average height and weight, and application of models to radio-frequency electromagnetic-field dosimetry," Physics in Medicine and Biology, vol. 49, no. 1, pp. 1-15, 2004.

[15] P. Dimbylow and W. Bolch, "Whole-body-averaged SAR from $50 \mathrm{MHz}$ to $4 \mathrm{GHz}$ in the University of Florida child voxel phantoms," Physics in Medicine and Biology, vol. 52, no. 22, pp. 6639-6649, 2007.

[16] E. Conil, A. Hadjem, F. Lacroux, M. F. Wong, and J. Wiart, "Variability analysis of SAR from $20 \mathrm{MHz}$ to $2.4 \mathrm{GHz}$ for different adult and child models using finite-difference timedomain," Physics in Medicine and Biology, vol. 53, no. 6, pp. 1511$1525,2008$.

[17] A. Hirata, I. Laakso, T. Oizumi, R. Hanatani, K. H. Chan, and J. Wiart, "The relationship between specific absorption rate and temperature elevation in anatomically based human body models for plane wave exposure from $30 \mathrm{MHz}$ to $6 \mathrm{GHz}$," Physics in Medicine and Biology, vol. 58, pp. 903-921, 2013.

[18] M. Cavagnaro, E. Pittella, and S. Pisa, "Anatomical models of breathing subjects for absorption and scattering analysis," in Proceedings of the International Symposium on Electromagnetic Compatibility, pp. 149-153, September 2013.

[19] K. S. Nikita, M. Cavagnaro, G. Cerri, S. Chiarandini, R. De Leo, and P. Russo, "A study of uncertainties in modeling antenna performance and power absorption in the head of a cellular phone user," IEEE Transactions on Microwave Theory and Techniques, vol. 48, no. 12, pp. 2676-2685, 2000.

[20] K. S. Kunz and R. J. Luebbers, The Finite Difference Time Domain Method For Electromagnetics, CRC Press, Boca Raton, Fla, USA, 1993.

[21] A. Taflove, Computational Electrodynamics: the Finite-Difference Time-Domain Method, Artech House, Norwood, Mass, USA, 1995.

[22] E. N. Marieb and K. Hoehn, Human Anatomy \& Physiology, Pearson International Edition, 2007.

[23] http://www.itis.ethz.ch/itis-for-health/tissue-properties/database/database-summary.

[24] A. Kraszewski and G. W. Hartsgrove, "A macroscopic model of lungs and a material simulating their properties at radio and microwave frequencies," Journal of Microwave Power and Electromagnetic Energy, vol. 21, no. 4, pp. 233-240, 1986.

[25] P. Gajšek, W. D. Hurt, J. M. Ziriax, and P. A. Mason, "Parametric dependence of SAR on permittivity values in a man model," IEEE Transactions on Biomedical Engineering, vol. 48, no. 10, pp. 1169-1177, 2001.

[26] A. Peyman, C. Gabriel, E. H. Grant, G. Vermeeren, and L. Martens, "Variation of the dielectric properties of tissues with age: the effect on the values of SAR in children when exposed to walkie-talkie devices," Physics in Medicine and Biology, vol. 54, no. 2, pp. 227-241, 2009.

[27] A. Christ, M. C. Gosselin, M. Murbach et al., "Age dependent changes in SAR and temperature distribution induced in the user's head by cellular phones," in BEMS Annual Meeting, p. 131, San Diego, Calif, USA, 2008.

[28] M. Cavagnaro, S. Pisa, and E. Pittella, "Safety aspects of people exposed to ultra wideband radar fields," International Journal of Antennas and Propagation, vol. 2013, Article ID 291064, 7 pages, 2013.

[29] S. Pisa, P. Bernardi, M. Cavagnaro, E. Pittella, and E. Piuzzi, "A circuit model of an ultra wideband impulse radar system for breath-activity monitoring," International Journal of Numerical Modelling: Electronic Networks, Devices and Fields, vol. 25, no. 1, pp. 46-63, 2012. 

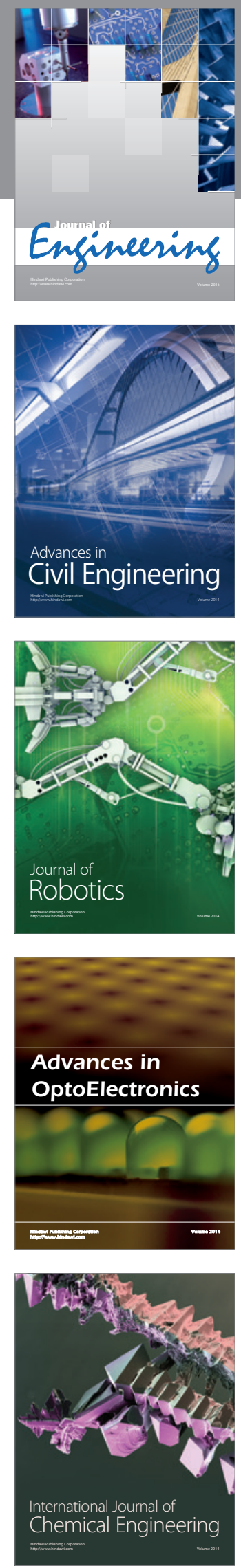

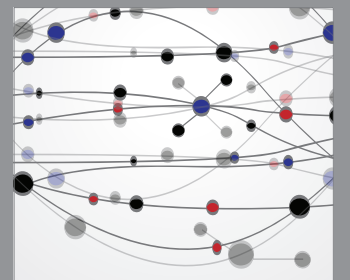

The Scientific World Journal
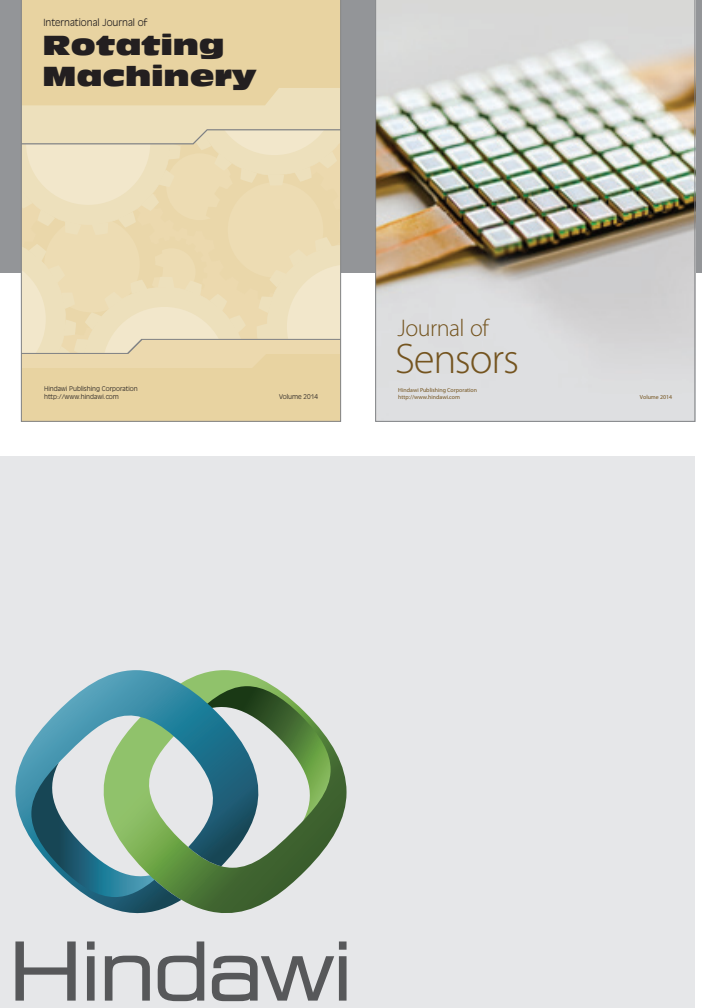

Submit your manuscripts at http://www.hindawi.com
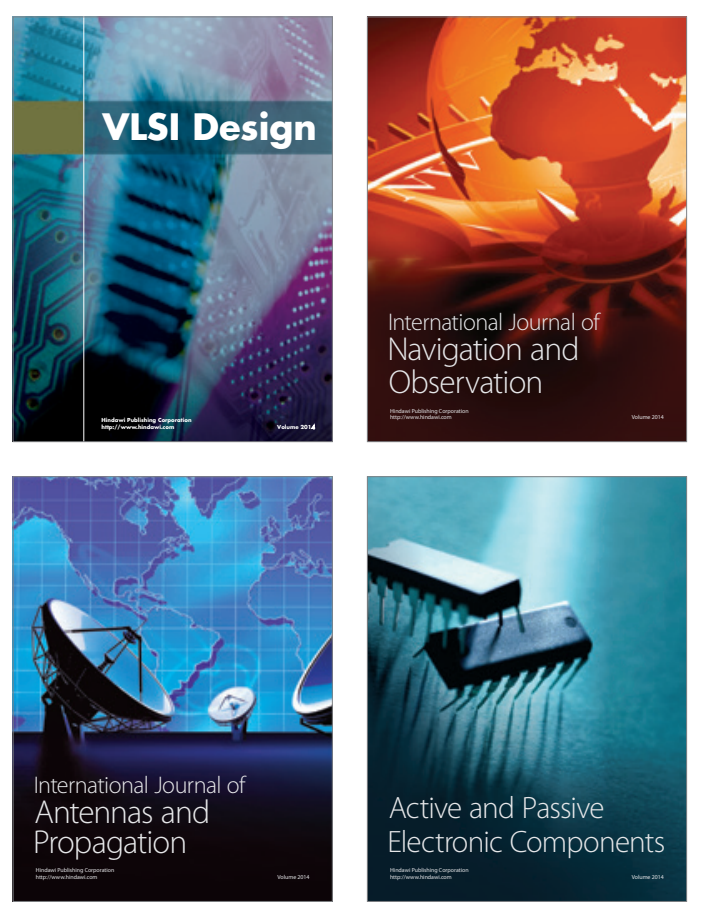
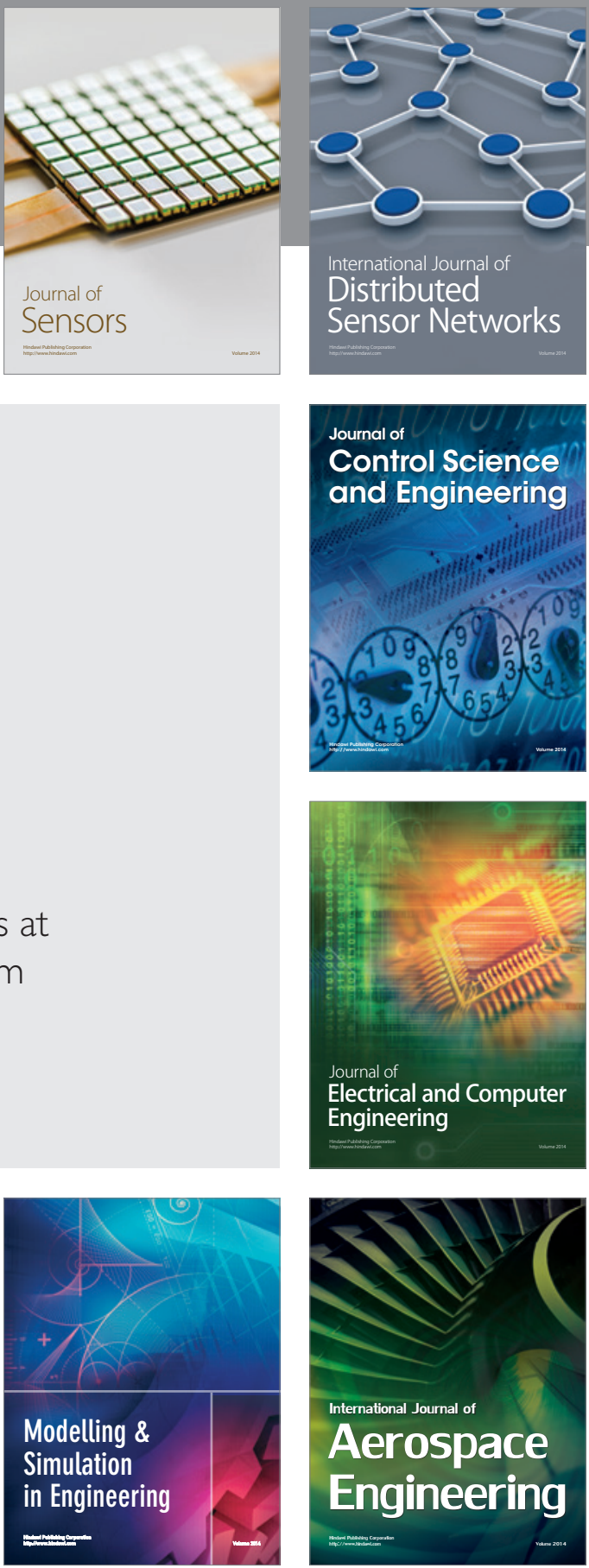

Journal of

Control Science

and Engineering
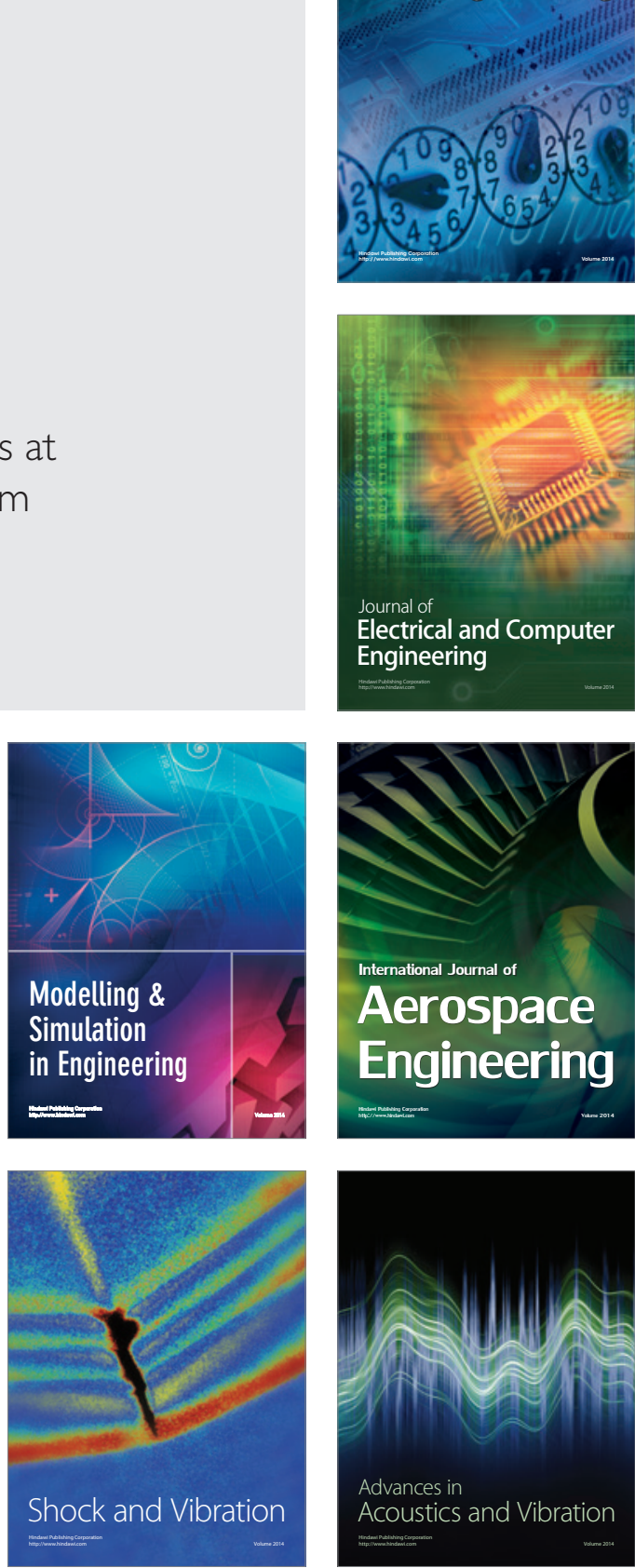